\title{
Mechanisms of Tumor-Induced Immunosuppression: Evidence for Contact-Dependent $T$ Cell Suppression by Monocytes
}

\author{
Michele L. Jaffe, Hiroshi Arai, and Gary J. Nabel \\ Departments of Internal Medicine and Biological Chemistry, \\ Howard Hughes Medical Institute, University of Michigan Medical \\ Center, Ann Arbor, Michigan, U.S.A.
}

\begin{abstract}
Background: The progressive growth of tumors in mice is accompanied by down-regulation of specific $\mathrm{T}$ cell responses. The factors involved in this suppression are not completely understood. Here, we have developed a model to examine the role of host immune effector cells in the inhibition of $\mathrm{T}$ cell function. In this model, progressive growth of a colon carcinoma line, CT26, is accompanied by loss of $\mathrm{T}$ cell response to alloantigens in both cytolytic and proliferation assays.

Materials and Methods: The CT26 tumor was inoculated into BALB/c syngeneic mice. Tumor growth, cytolytic $\mathrm{T}$ cell responses, lymphocyte proliferation, and flow cytometric analysis was performed in tumor-bearing animals 7 or 28 days after tumor inoculation.

Results: Spleen cells from tumor-bearing mice were found to suppress the proliferative response of spleen cells from normal mice to alloantigens. Examination
\end{abstract}

of the spleen cell population by FACS analysis revealed an increase in the percentage of monocytes as defined by expression of CDllb, the Mac- 1 antigen. Removal of the Mac-1-positive cells from the tumor-bearing hosts spleen relieved suppression of the tumor-bearing mouse spleen cell proliferative response to alloantigens, and addition of the Mac-1positive enriched cells suppressed proliferation of normal $\mathrm{T}$ cells in response to alloantigens. Cell contact was required for this inhibition.

Conclusions: Tumor induction of suppressive monocytes plays an important role in the general immunosuppression noted in animals bearing CT26 tumors. Identification of the mechanisms responsible for this effect and reversal of tumor-induced macrophage suppression may facilitate efforts to develop effective immunotherapy for malignancy.

\section{INTRODUCTION}

Large tumor burden is associated with suppression of the host's immune response to foreign antigens in both humans and animals $(1,2)$. Defects that occur in the specific immune response include both decreased proliferation and cytolytic activity $(3,4)$. Specific immunity is a consequence of $\mathrm{T}$ cells, through the $\mathrm{T}$ cell receptor (TCR), recognizing peptide fragments of foreign antigens in the context of self-major histocompatibility molecules while simultaneously receiv-

Address correspondence and reprint requests to: Gary J. Nabel, Departments of Internal Medicine and Biological Chemistry, Howard Hughes Medical Institute, University of Michigan Medical Center, 1150 West Medical Center Drive, Ann Arbor, MI 48109, U.S.A. ing other costimulatory signals (5). Both major histocompatibility antigens and the costimulatory signals exist on professional antigen-presenting cells (APC). These APC include monocytic and dendritic cells. The $T$ cell recognizes specific antigen in the context of a major histocompatibility antigen through its unique TCR. Once the TCR is engaged, signals are transduced into the cytoplasm through the $\mathrm{CD} 3$ complex, which is composed of six polypeptides, $\gamma \varepsilon, \delta \varepsilon$, and $\xi$ dimers (6). $\xi$ may also consist of homodimers or heterodimers with $\eta$ or FceRI $\gamma . \xi, \eta, \mathrm{CD} 3 \varepsilon$, and FceRI all have the ability to transduce signals independently into the cytoplasm (7). It is currently unclear as to the individual role of each of these polypeptides in signal transduction and $\mathrm{T}$ cell activation. 
Suppression of $\mathrm{T}$ cell responses to activation signals is also not completely understood. In murine tumor models, where suppression has been documented, studies suggest that suppressor $\mathrm{T}$ cells, suppressor macrophages, or suppressive cytokines secreted by tumor cells induce the impaired immune response in T cells $(4,8-10)$. Studies evaluating the suppressive effects of monocytic cells on $\mathrm{T}$ cells have suggested that either adherent macrophages $(10-13)$ or monocytes $(14,15)$ contribute to the decreased $\mathrm{T}$ cell response to activation signals and also decreased the expression of the $\xi$ chain, a major signaling protein in the TCR-CD3 complex; however, the role of soluble factors versus cellassociated gene products in mediating these effects is unclear. Studies demonstrate that while many soluble factors from monocytic cells contribute to the down-regulation of $\mathrm{T}$ cell responses to alloantigens (10), a contact-dependent signal is required to decrease the expression of the $\xi$ chain (15). The effects of contact-dependent signals delivered by monocytes to $\mathrm{T}$ cells on allogeneic responses is unknown.

We have investigated the mechanism of $\mathrm{T}$ cell suppression to alloantigens in a murine model of large tumor burden. In this model, cells of the monocytic lineage, as defined by expression of CD1 lb/Mac-1, gradually increase in number in the spleens of the mice as tumor size increases. In this paper, we describe the effects of these monocytes obtained by negative selection on $\mathrm{T}$ cells from both tumor-bearing and normal mice.

\section{MATERIALS AND METHODS} Mice

$\mathrm{C} 57 \mathrm{Bl} / 6 \mathrm{~J}\left(\mathrm{H}-2^{\mathrm{b}}\right)$ and $\mathrm{BALB} / \mathrm{c}\left(\mathrm{H}-2^{\mathrm{d}}\right)$ mice were used at 6 to 10 weeks of age and were obtained from Jackson Laboratories (Bar Harbor, ME, U.S.A.). Tumor-bearing mice (TBM) were injected subcutaneously with $8 \times 10^{5}$ cells of a single cell suspension of CT26, a syngeneic mouse colon adenocarcinoma that had been passed subcutaneously in BALB/C mice. Single cell suspensions of tumor were prepared by mixing minced tumor in Hanks' balanced salt solution (Sigma Chemical Co., St. Louis, MO, U.S.A.) with filtered DNAase $(0.025 \mathrm{mg} / \mathrm{ml}$, Sigma), hyaluronidase $(0.025 \mathrm{mg} / \mathrm{ml}$, Sigma) and collagenase $(0.025 \mathrm{mg} / \mathrm{ml}$, Sigma) with a stir bar for 1.5 $\mathrm{hr}$ at room temperature. Cells were then passed through Nytex and washed three times in Hanks' balanced salt solution.

\section{Cytolytic T Cell Assay}

Spleen cells from TBM or naive mice were incubated for 5 days with irradiated allogeneic spleen cells $\left(\mathrm{H}-2^{\mathrm{b}}\right)$ or $\mathrm{S} 194\left(\mathrm{H}-2^{\mathrm{d}}\right)$. On Day 5 , the lymphocytes were incubated in a cytolytic assay with radiolabeled $\left({ }^{51} \mathrm{Cr}\right.$ ) EL4 or YAC (MHC class $\mathrm{I}^{-}$) cells for $4 \mathrm{hr}$ at $37^{\circ} \mathrm{C}$ as described (16). Specific lysis is defined as [(experimental - spontaneous $\mathrm{cpm}) /($ total - spontaneous $\mathrm{cpm})] \times 100$.

\section{Proliferation Assay}

Spleen cells from TBM and/or normal mice were incubated for the indicated number of days with irradiated allogeneic spleen cells and irradiated syngeneic spleen cells or no cells, as controls, at the indicated ratios in 96 well dishes. On Day 4 , the cells were pulsed with $\left[{ }^{3} \mathrm{H}\right]$ thymidine for $16 \mathrm{hr}$ and cells were harvested on a Wallac Tomtec plate harvester and incorporation measured on a Wallac $\beta$ counter. Proliferation assays with membrane separations were performed in 6-well plates with cell separation across Costar's Transwell cell culture chamber inserts with $0.4 \mu \mathrm{M}$ pores. On Day 4, spleen cells were placed into 96-well dishes at the appropriate numbers and incorporation of $\left[{ }^{3} \mathrm{H}\right]$ thymidine measured. All assays were performed in RPMI 1640 media (Gibco, Gaithersburg, MD, U.S.A.) with $7.5 \%$ fetal calf serum, $2 \mathrm{mM}$ glutamine, $5 \times 10^{-5} \mathrm{M}$ $\beta$-2-mercaptoethanol, and penicillin/streptomycin and incubated at $37^{\circ} \mathrm{C}$ at $5 \% \mathrm{CO}_{2}$.

\section{Flow Cytometric Analyses}

Spleen cells from TBM and normal mice were blocked with an anti-Fc antibody and then stained with mAbs of appropriate specificities and analyzed on a FACScan flow cytometer (Becton Dickinson Immunocytometry systems, Mountain View, CA, U.S.A.) after gating out dead cells in Fl2 by using propidium iodide staining. The following monoclonal antibodies (mAbs) were used: rat anti-mouse CD32/16 (2.4G2), FITClabeled rat anti-mouse CD90/Thy-1.2 (53-2.1), FITC-labeled rat anti-mouse CD45R/B220 (RA36B2), FITC-labeled rat anti-mouse CD 1 lb/Mac- 1 $(\mathrm{Ml} / 70)$, FITC-labeled rat IgG2b, $\kappa$, and FITClabeled rat IgG2a, $\kappa$. All antibodies were obtained from Pharmingen (San Diego, CA, U.S.A.) and used at a concentration of $1 \mu \mathrm{g} /$ million cells. For Fas and FasL immunostaining of $\mathrm{T}$ and monocytic cells, the following antibodies were used: Fas, hamster anti-mouse Fas (clone VC8-4B3) 
with FITC-labeled mouse anti-hamster IgG (clones G70-204 and G94-56) (Pharmingen); FasL, mouse Fas-human $\gamma_{1}$ Fc fusion protein with FITC-labeled goat anti-human IgG Fc (Jackson Immunoresearch Lab, West Grove, PA, U.S.A.); Mac-1 (CDIlb), phycoerythrin (PE-labeled rat anti-mouse CDl7b (clone M1/70.15) (Caltag, Burlingame, CA, U.S.A.); Thy-1, PE-labeled rat anti-mouse CD90 (Caltag); and control, PE-labeled rat IgG2b (Caltag).

\section{Cell Depletion}

Spleen cells from TBM were depleted of $T$ cells, B cells, and monocytes by pretreatment with rat antibody specific for the surface antigens and then removed with the use of magnetic beads coated with sheep anti-rat IgG. The following primary mAbs were used: rat anti-mouse CD90/Thy-1.2 (53-2.1), rat anti-mouse CD45R/ B220 (RA3-6B2), rat anti-mouse CD1 lb/Mac-1 $(\mathrm{Ml} / 70)$, all rat IgG2a, $\kappa$. All antibodies were obtained from Pharmingen. Coated cells were then washed twice and incubated with Dynabeads M-450 sheep anti-rat IgG (Dynal, Oslo, Norway) at a bead-to-cell ratio of $40: 1$ at $4^{\circ} \mathrm{C}$ for $30 \mathrm{~min}$ on a rocker. Magnetic beads attached to cells were then removed from unattached cells with a Dynal MPC-6 magnet. Unattached cells were washed once, counted, and added to assays at appropriate ratios. Depletions were $>98 \%$ for all cell types as measured by flow cytometric analyses.

\section{RESULTS}

\section{Inhibition of $T$ Cell Cytotoxic and Proliferative Responses in Spleens from Late Tumor-Bearing Mice in Vitro}

To evaluate $\mathrm{T}$ cell function in mice at early and late time periods after bearing tumors, cytolytic $\mathrm{T}$ cell assays were performed after appropriate in vitro sensitization. Allogeneic and NK responses were determined by a chromium release assay using either an allogeneic tumor cell line, EL4, or a class I-deficient cell line, YAK, as targets. In four separate experiments, lysis of both class $\mathrm{I}^{+}$ and class $\mathrm{I}^{-}$targets was substantially reduced when $\mathrm{T}$ cells were derived from the spleen of late TBM, in contrast to normal controls (Fig. $1 \mathrm{~A}$ and $\mathrm{B}$, right panel). In contrast, lysis of both class $\mathrm{I}^{+}$ and class $\mathrm{I}^{-}$targets was comparable to normal controls when cells were derived from early TBM
(Fig. 1A and B, left panel), and these results were confirmed in at least two separate experiments.

In addition to its decreased cytolytic activity, the yield of cells from the late tumor-bearing mouse spleen was reduced compared with normal mouse spleen after in vitro sensitization. This finding suggested a decreased ability of the spleen cells from late TBM to proliferate in response to allogeneic stimulation. To test this hypothesis, proliferation was measured after stimulation with irradiated allogeneic spleen cells. Under conditions that allowed significant proliferation of normal mouse lymphocytes, minimal proliferation was detected in late TBM lymphocytes (Fig. 1C). Similar effects were observed at all time points (Days 3-6) at different responderto-stimulator cell ratios (data not shown).

\section{Spleens from Tumor-Bearing Mice Have a Higher Percentage of Monocytes, as Defined by the CD11b/Mac-1 Antigen, than Spleens from Normal Mice}

To define the cell types that mediated the change in $\mathrm{T}$ cell responses, spleen cell suspensions from normal and late tumor-bearing mice were analyzed by flow cytometry with antibodies to mouse CD90/Thy-1.2, CD45R/B220, and CD1lb/Mac-1 (Fig. 2). In late tumor-bearing mice, the percentage of CD90/Thy-1.2-positive spleen cells changed slightly from 31 to $27 \%$ of total cells, and the percentage of CD45R/B220-positive spleen cells decreased from 41 to $30 \%$. In contrast, the percentage of CD1 1b/Mac-1-positive spleen cells increased from 2 to $15 \%$. Although some subsets of cytolytic T cells express CDIlb, it is unlikely that this subset represented splenic $\mathrm{T}$ cells, because the population of expanded $\mathrm{CD} 11 \mathrm{~b}^{+}$cells showed a larger size as measured by forward light scatter than the subsets staining with Thy-1.2 antibody. In addition, cells that expressed the Mac- 1 antigen also coexpressed small amounts of Mac-2 antigen, another marker of cells of monocytic lineage (data not shown). The minor shift in the $\mathrm{T}$ cell population suggested that the change in responsiveness of $\mathrm{T}$ cells was qualitative rather than quantitative. This small decrease in percentage would be unlikely to account for the large decrease in proliferation when stimulated. However, the larger shifts in the B cell and monocyte populations suggested that tumor growth was influencing their growth and survival to a greater extent in vivo. 
EARLY

A.

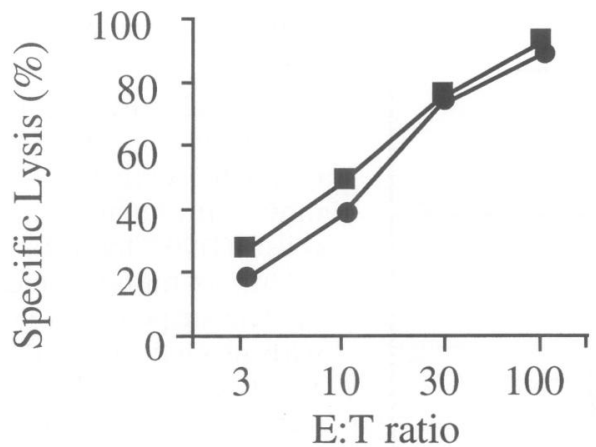

B.
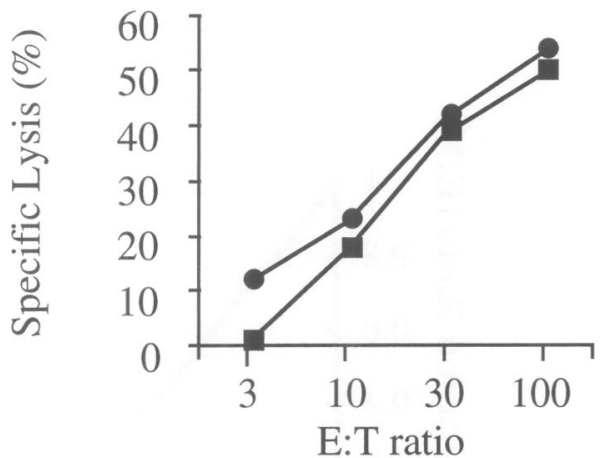

LATE
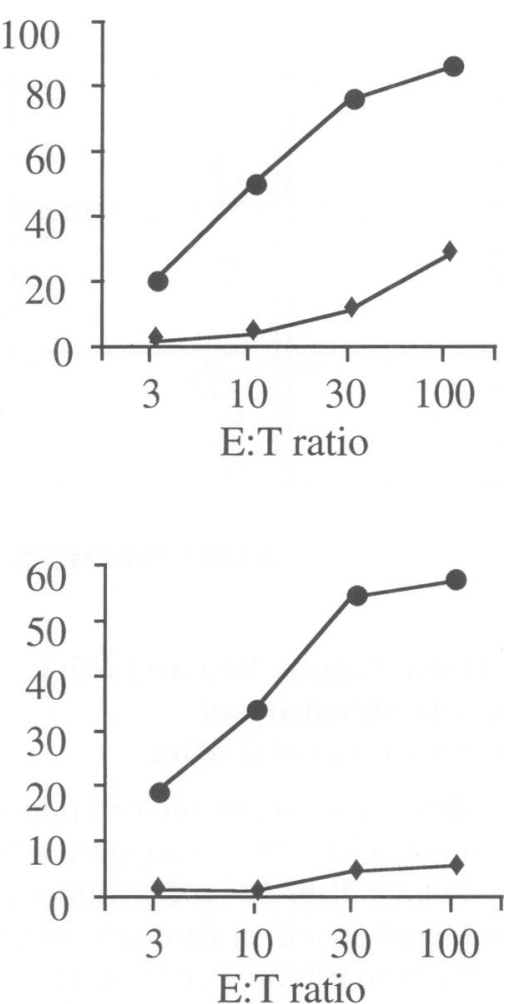

C.

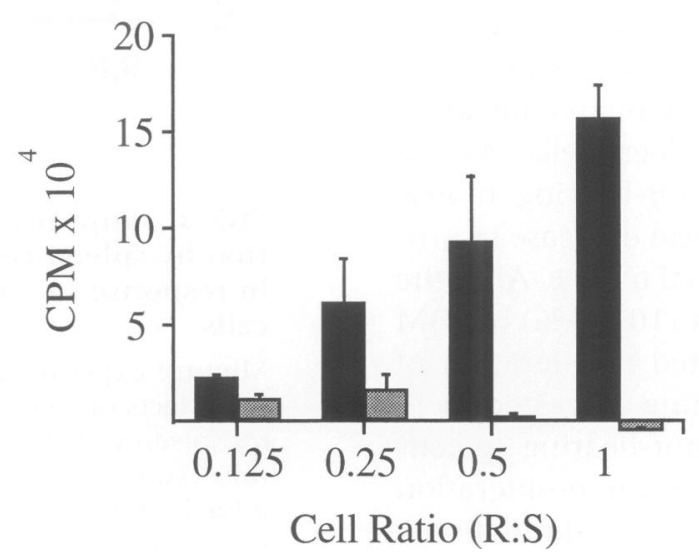

FIG. 1. Suppression of cytolytic activity in spleen cells of tumor-bearing mice against targets with (A) and without $(B)$ the expression of class I molecules and suppression of proliferation in response to alloantigens (C)

(A) Spleen cells from normal mice ( $\bullet$ ), early tumor-bearing mice ( $<28$ days) ( $\square$ ), or late tumor-bearing mice ( $>28$ days) ( $)$ of BALB/c origin were incubated with irradiated stimulator cells for 5 days and then mixed with ${ }^{51} \mathrm{Cr}$-labeled target cells at different effector:target ratios in a $4-\mathrm{hr}{ }^{51} \mathrm{Cr}$ release assay. (A) Allogeneic spleen cells from C57Bl/6 mice were used as stimulators and EL4 cells were used as targets. (B) Syngeneic S194 cells were used as stimulators and YAC cells were used as targets. Values are the mean of triplicate wells. Experiments are representative of at least four different experiments with a total of six early tumor-bearing mice and six late tumor-bearing mice. (C) Responder spleen cells from normal mice (BALB/c) ( $\square$ ) or from late tumor-bearing mice (>28 days) (BALB/C) (圈), were incubated with allogeneic stimulator cells $(\mathrm{C} 57 \mathrm{Bl} / 6)$ at the indicated responder: stimulator (R:S) cell ratios. The stimulator cell number was held constant at $4 \times 10^{5}$ cells/well and the responder cell number was varied. $\left[{ }^{3} \mathrm{H}\right]$ was added on Day 4 of culture and incorporation was measured $16 \mathrm{hr}$ later. Results are reported as CPM \pm SEM from cells stimulated by allogeneic spleen cells minus CPM from cells stimulated by syngeneic spleen cells. Values are the mean CPM from triplicate wells. 


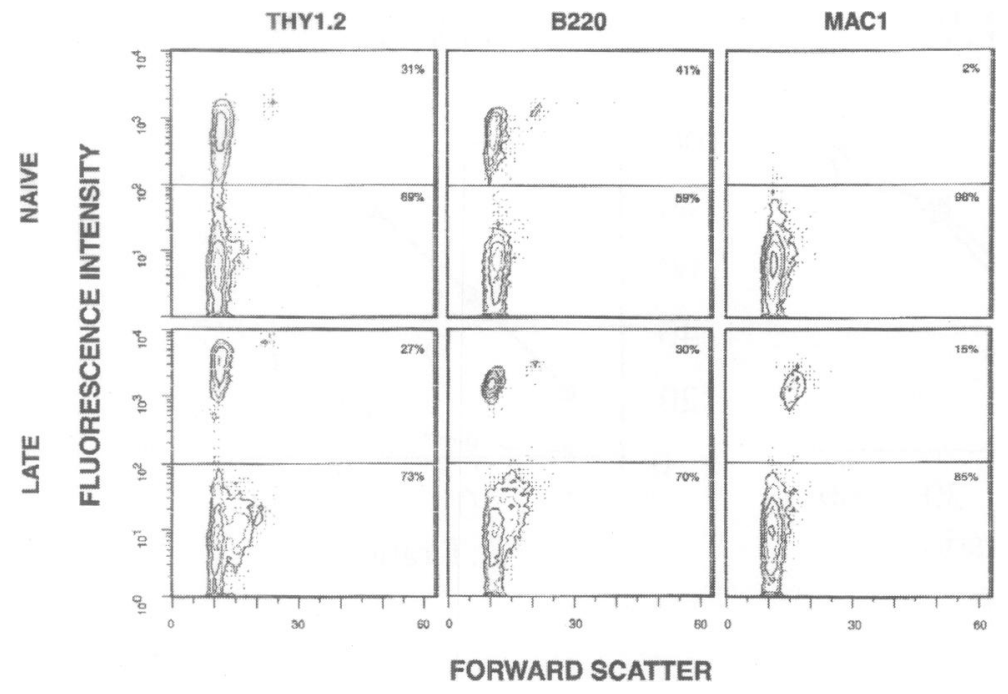

FIG. 2. Increased percentage of monocytes as defined by expression of the CDIlb/Mac-l antigen in spleens from late tumor-bearing hosts

Spleen cell suspensions from naive mice and from late tumor-bearing mice were incubated with FITC-labeled rat antimouse antibodies that detect primarily $\mathrm{T}$ cells (CD90/Thy-1.2), B cells (CD45R/ B220), or monocytic cells (CD l lb/Mac1). The suspensions were then treated with propidium iodide and analyzed on a FACscan after dead cells were gated out in FL2. The abscissa is forward scatter; the ordinate is fluorescence intensity of the FITC label. The percentage of cells staining positive is given in the upper half in each graph.

\section{Spleen Cells from Tumor-Bearing Mice Can Suppress Proliferation of Lymphocytes from Normal Mice}

The change in phenotype of splenic cell populations in the tumor-bearing spleens suggested that the tumor may not act directly on T lymphocytes but may instead exert its effect through another immune effector cell to inhibit $\mathrm{T}$ cell function. To explore this possibility, mixture experiments were performed. Different ratios of normal spleen cells and TBM spleen cells were used in a standard proliferation assay, and results were compared with the proliferation of an equivalent number of normal mouse spleen cells. As the number of cells from a tumor-bearing mouse increased, there was a reciprocal decrease in proliferation by cells from a normal mouse. Also, the addition of a small proportion (10-30\%) of TBM spleen cells markedly inhibited proliferation of normal cells (Fig. 3). This finding suggested there was a cell population in tumor-bearing spleens that could act in trans to inhibit the proliferation of $\mathrm{T}$ cells in response to allogeneic stimulation.

\section{Depletion of $\mathrm{CD}_{11 b^{+}}$Cells from Tumor- Bearing Mouse Spleen Restores Proliferation of the Lymphocytes in Response to Alloantigens}

To identify the specific cell type responsible for the decreased allogeneic response, cell depletion experiments were performed. Different cell populations were removed from tumor-bearing mouse spleen by coating the cells with specific antibodies to either CD90/Thy-1.2, CD45R/ $\mathrm{B} 220$, or CD1lb/Mac-1 antigen, and these cells

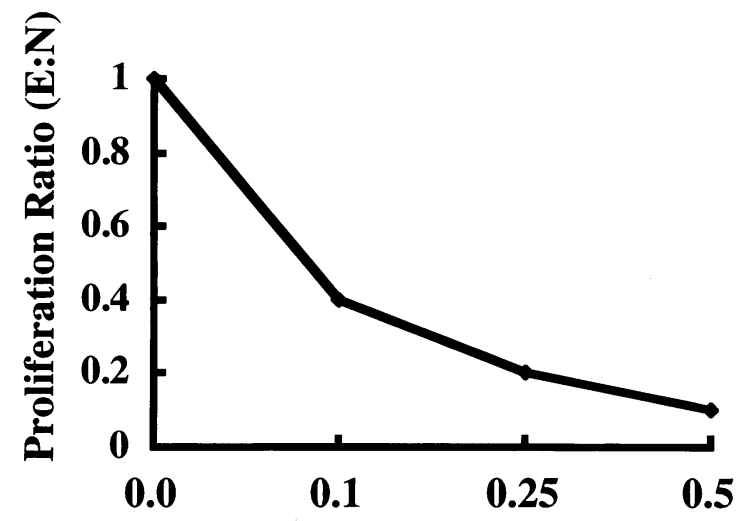

Cell Fraction (TB/T)

FIG. 3. Suppression of lymphocyte proliferation by spleen cells from tumor-bearing mice in response to stimulation by allogeneic spleen cells

Mixture experiments were performed to examine the effects of spleen cells from tumor-bearing mice on spleen cells from normal mice. For the proliferation assay, spleen cells from a normal mouse were mixed with spleen cells from a late tumor-bearing mouse. $4 \times 10^{5}$ stimulator cells $(\mathrm{C} 57 \mathrm{Bl} / 6)$ and $4 \times$ $10^{5}$ responder cells (BALB/c) were incubated together in a 96-well plate in triplicate wells. [ $\left.{ }^{3} \mathrm{H}\right]$ was added on Day 4 of culture and incorporation was measured $16 \mathrm{hr}$ later. The cell fraction is the number of cells from the tumor-bearing mouse spleen (TB) divided by the total number of spleen cells (T) (tumorbearing plus normal spleen cells). The proliferation ratio is the mean CPM from triplicate wells containing mixtures of cells from normal and tumor-bearing mouse spleens (E) divided by the mean CPM from wells containing equivalent numbers of normal cell (N). Control spleen cells stimulated with irradiated syngeneic spleen cells demonstrated less than 10 times the amount of stimulation as the experimental group of spleen cells. Experiments are representative of results from at least three independent experiments. 
were removed with the use of magnetic beads coated with anti-immunoglobulin. The remaining cells were analyzed by using a standard allogeneic proliferation assay. Depletion of CD1 lb/ Mac-1-positive cells restored the ability of TBM spleen cell to proliferate in response to an allogeneic stimulus (Fig. 4A). In contrast, depletion of CD45R/B220 cells, largely composed of B cells, resulted in a small loss of suppression and was much less effective than removal of $\mathrm{CDIlb} /$ Mac-1 cells (Fig. 4A). As expected, no proliferation was observed after CD90/Thy-1.2-positive cell depletion or with a nonspecific control antibody (Fig. 4A). Although $\mathrm{T}$ cells demonstrated increased proliferation to allogeneic stimuli when the monocytes were removed, proliferation did not increase to levels of control normal mouse spleen cells.

To determine whether the $T$ cell could act in trans on normal $\mathrm{T}$ cells, mixing experiments were performed with tumor-bearing spleen cells depleted or enriched for T cells (Fig. 4B). CD90/ Thy-1.2-depleted TBM spleen cells inhibited normal mouse spleen cells at a 9:1 ratio to the same extent as TBM cells without depletions (Fig. 4B). Enrichment of T cells by CD45R/B220 and CD1lb/Mac-1 depletion demonstrated a greater proliferative response than nondepleted cells (Fig. 4B). Therefore, it appeared that the CDIlb/Mac-1 cell from TBM was responsible for the major inhibitory signals to $\mathrm{T}$ cells.

\section{Cell Contact Is Required for Inhibition of Naive Spleen Cell Responses to Alloantigens by the CD90/Thy-1.2- CD45R/B220 ${ }^{-}$Cell Population from Tumor-Bearing Mouse Spleen}

To determine whether cell contact or soluble factors mediated this effect, inhibition by the CDIlb/Mac-1 cell was analyzed further by supernatant transfer and membrane separation experiments. Transfer of supernatant from CD90/ Thy-1.2 and CD45R/B220 depleted TBM spleen failed to confer suppression upon normal spleen cells (Fig. 5A). Also, the addition of neutralizing antibodies to mouse transforming growth factor- $\beta$ (TGF- $\beta$ ), interleukin 10 (IL-10), and tumor necrosis factor $\alpha$ (TNF $\alpha$ ) or indomethacin, which inhibits $\mathrm{PGE}_{2}$ production, to proliferation assays using TBM spleen did not change T cell proliferation (data not shown). In contrast, cell contact conferred this inhibition (Fig. 5A).

To confirm these results, experiments were also performed with cells of the TBM separated from normal mouse spleen by a membrane in a proliferation assay. When the cells were separated, there was no inhibition of proliferation, but when cell contact was allowed there was significant inhibition of proliferation of the normal mouse cells (Fig. 5B). Although isolated cell membranes did not confer suppression, high concentrations of glutaraldehyde-fixed cells did mediate the effect, suggesting that intact cells and continued protein synthesis are required for optimal immunosuppression. This data suggests that either a membrane bound or a cell-associated factor mediates inhibition of the $\mathrm{T}$ cell in response to activation signals.

Analysis of monocytic cells derived from primary tumors was performed. Cell suspensions were prepared after digestion with collagenase and elastase, and immunofluorescence analysis was performed. The expression of Fas ligand was found to be elevated in monocytes derived from tumor cells (Fig. 6B), in contrast to monocytic cells described previously from spleen and other lymphoid organs. No significant increased expression of Fas or FasL was detected in T cells, although a small ( $\sim 3$-fold) increase in $\mathrm{CD} 3 \mathrm{ex}-$ pression was noted in these cells (data not shown). These data raise the possibility that Fas ligand may contribute to the immunosuppression induced in tumor-bearing animals.

\section{DISCUSSION}

We have demonstrated a quantitative increase in monocytes which inhibit $\mathrm{T}$ cell proliferation and cytotoxicity in mice with large burdens of a subcutaneously implanted tumor, CT26. Decreased immune function was observed in animals with a large tumor burden. Cytolytic and proliferative $\mathrm{T}$ cell responses to alloantigen and natural killer (NK) activity were markedly diminished. Increased levels of monocytic cells were detected in the spleen of these animals, and we find that this cell type was responsible for the reduction in $\mathrm{T}$ cell response. Finally, we have found that contact between $\mathrm{T}$ cells and monocytes was required for this inhibitory effect.

This study provides insight into the mechanism of tumor-induced immunosuppression. Defects in the immune response of tumor-bearing animals have been attributed to soluble factors produced by tumor cells, which act directly on $\mathrm{T}$ cells or indirectly, through the induction of other suppressor cells, either $\mathrm{T}$ cells or macrophages $(4,8-10)$. In the animal model described in this 
study, it was found that the monocyte conferred suppressive activity on the $\mathrm{T}$ cell. Other groups who have found monocytic cells to be suppressive have shown that soluble factors, including PGE $_{2}$ and TGF- $\beta$, were important in the suppression of lymphocyte proliferation (11-14). In contrast, we found that cell contact was required to inhibit $\mathrm{T}$ cell function. These differences could be accounted for by a difference in tumor type or, alternatively, by the methods used to obtain the monocytes. Studies that have found soluble factors to be important obtained monocytic cells by separating cells on the basis of adherence, which may induce activation and enhance cytokine secretion. These effects could mask the additional inhibitory effects of cell surface molecules. In the present study, cells were derived by enrichment
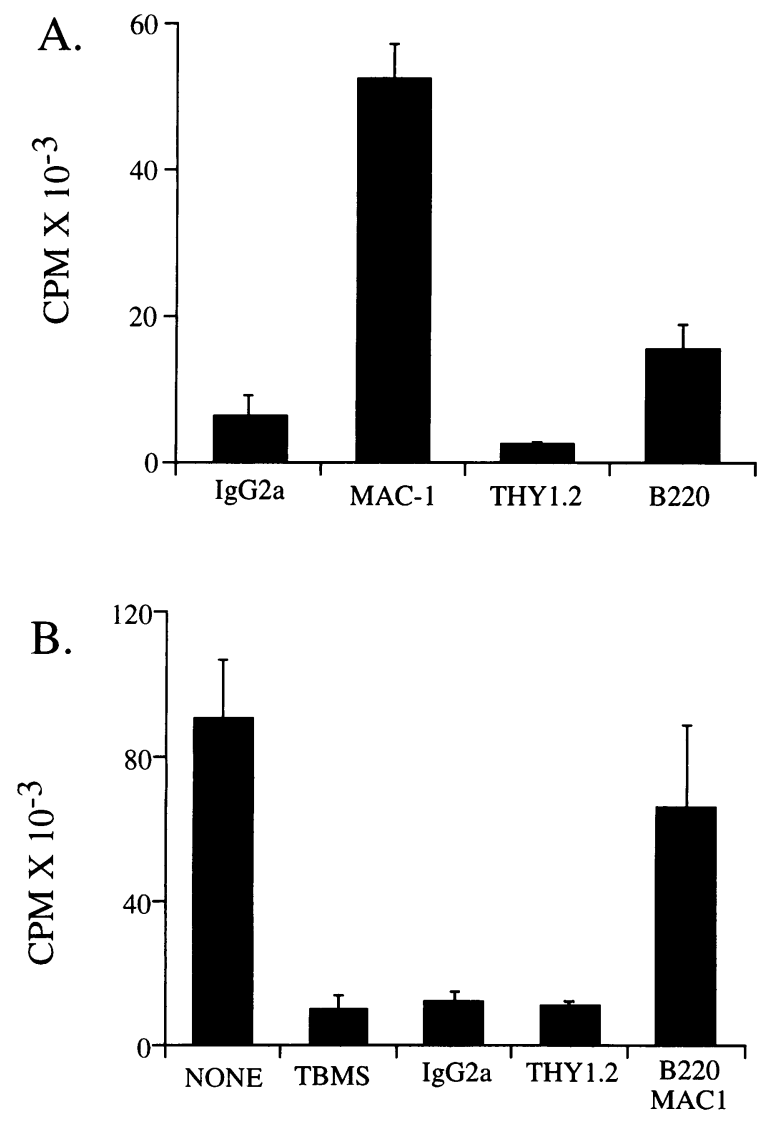

through the negative selection of $\mathrm{T}$ and $\mathrm{B}$ cells. When a positive selection by Mac- 1 and Mac- 2 antigens or depletion of $\mathrm{T}$ and $\mathrm{B}$ cells were used to isolate monocytes, however, no evidence of soluble inhibitors of $\mathrm{T}$ cell function was detected $(14,15)$, consistent with findings reported here.

There is both direct and indirect evidence to suggest that tumor induction of suppressive monocytes may represent a general mechanism of tumor-induced immunosuppression. First, several groups using different tumor lines have noted an increase in the percentage of monocytes in the spleen of late tumor-bearing animals $(10,12,17)$. Second, several different cell lines have quantitative changes in the $\zeta$-chain of the TCR complex, which is induced by cell contact with monocytes derived from tumor-bearing an-

FIG. 4. Restoration of lymphocyte proliferation in spleen cells from tumor-bearing mice depleted of cells with Mac-l surface antigen in response to stimulation by allogeneic spleen cells

(A) Spleen cells were incubated with the primary rat anti-mouse antibodies IgG2a (control), CD1 lb/Mac-1, CD90/ Thy-1.2, or CD45R/B220. The cells were washed and incubated with magnetic beads coated with anti-rat antibody. After a 30-min incubation, the magnetic beads with attached cells were removed. The nondepleted cells incubated with the control antibody were brought up to $4 \times 10^{6}$ cells $/ \mathrm{ml}$. and the other groups were brought up to equivalent volumes. One hundred microliters of these cells were then incubated with $4 \times 10^{5}$ irradiated allogeneic stimulator cells. $\left[{ }^{3} \mathrm{H}\right]$ thymidine was added on Day 4 of culture and incorporation was measured $16 \mathrm{hr}$ later. Results are reported as CPM \pm SEM. Values are the mean CPM from triplicate wells. Experiments are representative of results from at least three independent experiments. (B) Mixing experiments were performed to evaluate the effects of CD90/Thy-1.2 positive spleen cells derived from TBM on naive spleen cells. Responder spleen cells from naive mice were incubated alone, with TBM spleen cells, or with TBM spleen cells depleted of cells with IgG2b, CD90/Thy-1.2, or CD1 lb/Mac-1 and CD45R/B220 primary antibodies and magnetic beads coated with secondary antibodies. The wells contained $3.6 \times 10^{5}$ naive spleen cells. The TBM spleen cells and TBM spleen cells incubated with the control antibody were brought up to $3.6 \times 10^{6}$ cells $/ \mathrm{ml}$ and the other groups were brought up to equivalent volumes. One hundredth of a milliliter of these cells were added to the normal spleen cells. The mixtures were then incubated with $4 \times 10^{5}$ irradiated allogeneic stimulator cells. $\left[{ }^{3} \mathrm{H}\right]$ thymidine was added on Day 4 of culture and incorporation was measured $16 \mathrm{hr}$ later. Results are reported as CPM \pm SEM. Values are the mean CPM from triplicate wells. Control responder cells from normal mouse spleen without stimulation demonstrated less than 2.5 times the amount of stimulation as the naive mouse spleen cells stimulated with irradiated allogeneic spleen cells. 
A.

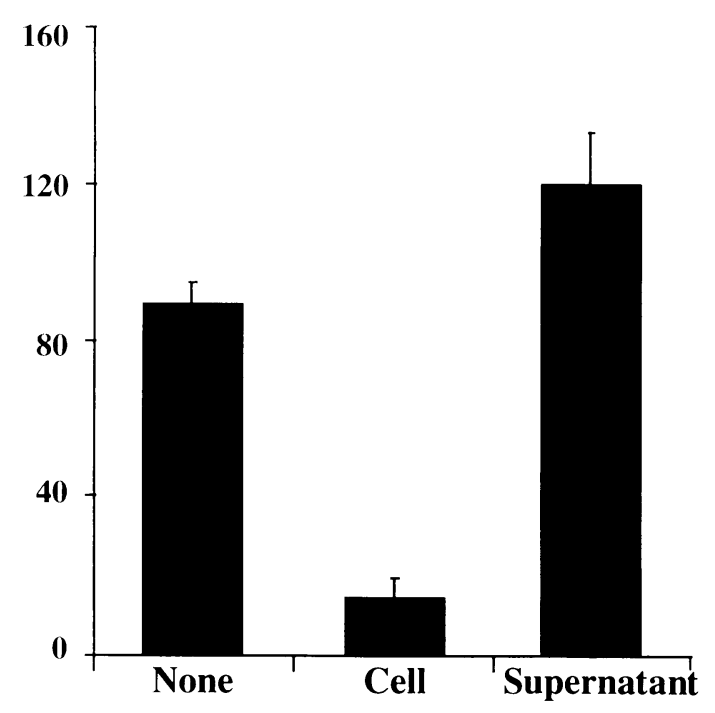

B.

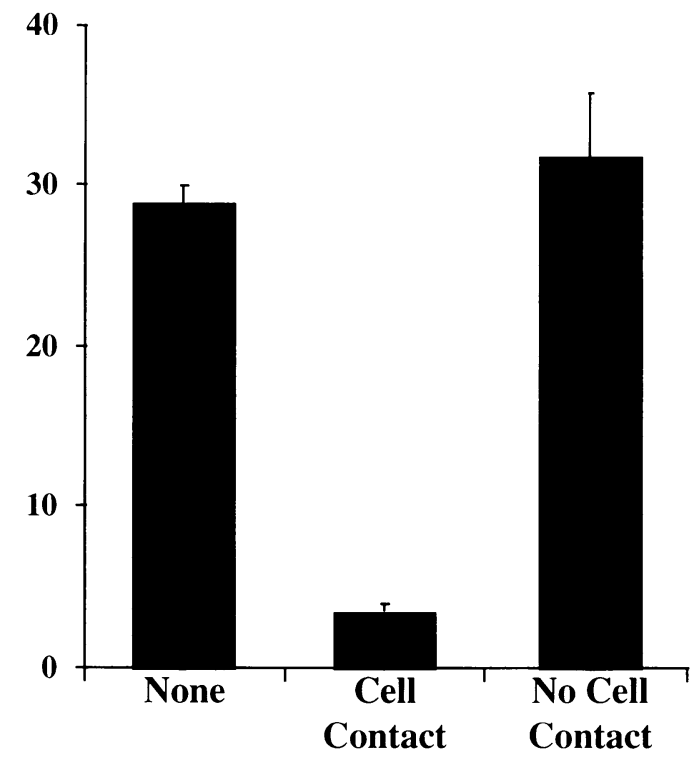

FIG. 5. Inhibition of lymphocyte proliferation by cells from tumor-bearing mouse spleen expressing the $C D 1$ lb/Mac-1 surface antigen requires cell contact

(A) Mixing experiments were performed to evaluate inhibition by cells or soluble factors. Responder spleen cells from naive mice were incubated with either CD90/Thy-1.2, CD45R/B220- spleen cells (80\% CD1 lb/Macl positive by FACS analyses) from tumor-bearing mice or supernatant after incubation for 24 hr at a 9:1 ratio in 96-well plates. These treated responder cells were then incubated with an equivalent number of irradiated allogeneic stimulator cells. ['H]thymidine was added 4 days later and incorporation was measured after an additional $16 \mathrm{hr}$. Results are reported as mean CPM \pm SEM from triplicate wells. Experiments are representative of results from at least three independent experiments and at a 5:1 ratio. (B) Membrane separation was performed to evaluate inhibition by soluble factors. Responder spleen cells from naive mice were incubated with spleen cells from tumorbearing mice at a 3:1 ratio in the same well or separated by a semipermeable membrane. The mixtures were then incubated with an equivalent number of irradiated allogeneic stimulator cells. $\left[{ }^{3} \mathrm{H}\right]$ thymidine was added 4 days later and the cells were placed into wells in a 96-well dish and incorporation was measured after an additional 16 hr. Results are reported as mean CPM \pm SEM from triplicate wells.

imals $(15,18)$. Third, monocytes from animals bearing the DI-DMBA-3 mammary adenocarcinoma induces suppression of naive $\mathrm{T}$ cells by a contact-dependent manner in response to Con A (14).

The mechanism by which tumor cells induce a suppressive phenotype in monocytes is currently unknown. It is possible that tumor cells provide signals to these immune cells by two mechanisms. First, tumor cells have been demonstrated to elaborate a variety of cytokines, and these could induce the monocyte to become suppressive. Such a cytokine would induce the monocyte to deliver signals to the $\mathrm{T}$ cell which inhibit the response to activation signals. Although it is possible that such cytokines could affect antigen presentation, such a defect would not likely be responsible for the inhibition observed here, since antigen-presenting cells reside in the allogeneic irradiated stimulator population and provide costimulatory signals in our experiments. While there would be many candidate cytokines that would prevent antigen presentation to the $\mathrm{T}$ cell and that are produced by tumors, such as IL- 10 and IL- 1 receptor antagonist $(19,20)$, no soluble factors are known that induce the monocytic phenotype noted in our studies. Alternatively, chronic stimulation of the immune system by large amounts of tumor antigens may alter TCR and cytokine signaling through immune cell surface interactions. In support of this theory, it has been shown that antibody to CDI4 on human monocytes induces a contact-dependent signal to $T$ cells, which inhibits proliferation in response to activation signals. It has been proposed that a CDl4 ligand might exist on activated $\mathrm{T}$ cells which interacts with CDl 4 on monocytes as part of a negative regulatory circuit (21). Although the molecule induced on the monocyte is not known, the find- 
A.

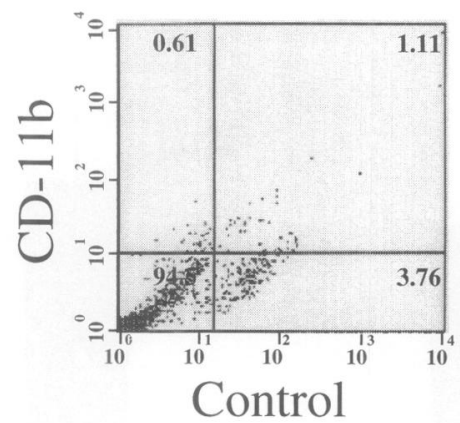

B.

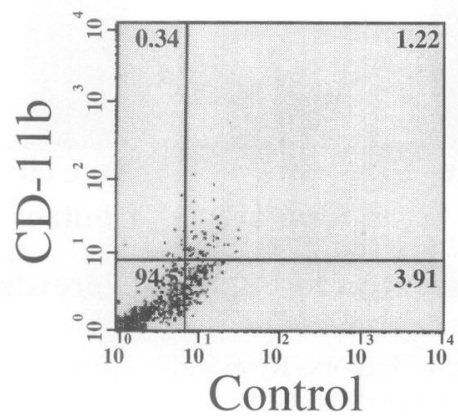

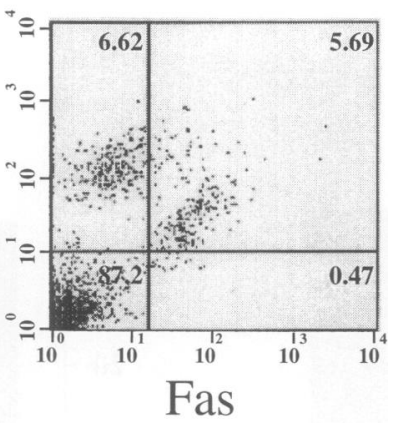

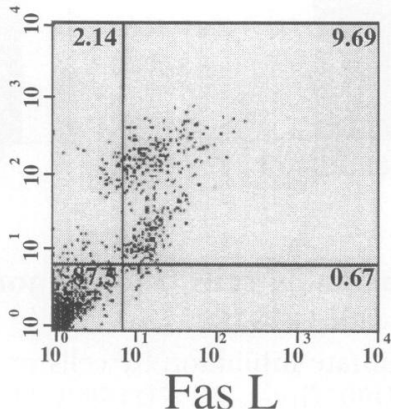

FIG. 6. FACS analysis of Fas and Fas ligand expression in monocytic cells derived from tumors

Cell suspensions were prepared from tumor-bearing animals (Day 18) and incubated with antibodies directed to Mac-1 (ordinate) and Fas (A) or Fas ligand (B). Negative control antibody immunofluorescence is shown at the right on each panel as indicated. The abscissa shows staining for Fas or Fas ligand as indicated, and the percentage of positive cells for each antibody is shown in the upper panel of the relevant regions of analysis. ing of increased FasL expression in this population suggests its potential involvement in this effect. It may also provide a useful surrogate marker for tumor-induced immunosuppression.

The molecular signal from monocyte to $\mathrm{T}$ cell in this study is membrane or cell associated. Candidate molecules could include TGF- $\beta$ and nitric oxide, as they have been demonstrated to decrease proliferation of activated lymphocytes and can be associated with the membrane (TGF- $\beta$ ) (22) or lose activity quickly after being released from the cell (nitric oxide) (23). In some instances, TGF- $\beta$ antibodies can reverse inhibition by monocytes, for example, in hyperimmunized mice (22), however, neither of these molecules has been demonstrated to decrease $\xi$ expression in the TCR, and neutralizing antibodies to TGF- $\beta$ did not block the negative signal to $\mathrm{T}$ cells in this study (data not shown). These findings suggest that TGF- $\beta$ is not involved in decreasing $\mathrm{T}$ cell proliferation in our system.

The CDI lb/Mac- ${ }^{+}$monocytic cell from tumor-bearing mice has been demonstrated to down-regulate $\mathrm{T}$ cell responses to alloantigens. This data, in conjunction with recent studies that demonstrate that $\mathrm{CD} 1 \mathrm{lb} / \mathrm{Mac}^{+} \mathrm{1}^{+}$cells downregulate expression of the $\xi$-chain of the $\mathrm{CD}_{3}$ -
TCR complex supports the hypothesis that a $\mathrm{CD} / \mathrm{lb} / \mathrm{Macl}^{+}$cell-associated factor plays an important role in determining responsiveness to antigens in animals with a large tumor burden. The monocyte, which in some instances can provide appropriate peptide fragments and costimulatory molecules to activate specific $\mathrm{T}$ cells, could provide inhibitory signals to prevent $\mathrm{T}$ cell proliferation in response to appropriate activation signals. The capacity to inhibit such $\mathrm{T}$ cell responses may be an important control mechanism not only in tumor-induced evasion of immune responses, but also for appropriate recognition of viral proteins and auto-antigens.

\section{ACKNOWLEDGMENTS}

We would like to thank Donna Gschwend for secretarial assistance, Karen Carter for preparation of figures, Judy Stein for help in figure preparation and statistical analysis, and Dr. Keith Bishop for helpful advice and discussion. This work was supported in part by a grant from the National Institutes of Health (P01 CA59327-01; $\mathrm{NIH}$. 


\section{REFERENCES}

1. Broder S, Waldmann TA. (1978) The suppressor network in cancer. N. Engl. J. Med. 299: 1281-1284.

2. Deckers PJ, Davis RC, Parker GA, Mannick JA. (1973) The effect of tumor size on concomitant tumor immunity. Cancer Res. 33: 139.

3. Maccubbin DL, Mace KF, Ehrke MJ, Mihich E. (1989) Modification of host antitumor defense mechanisms in mice by progressively growing tumor. Cancer Res. 49: 4216-4224.

4. North RJ, Bursuker I. (1984) Generation and decay of the immune response to a progressive fibrosarcoma. J. Exp. Med. 159: 1312-1321.

5. Janeway CA, Golstein P. (1993) Lymphocyte activation and effector functions. Curr. Opin. Immunol. 5: 313-323.

6. Weiss A. (1993) T cell antigen receptor signal transduction: A tale of tails and cytoplasmic protein-tyrosine kinases. Cell 73: 209-212.

7. Weiss A, Littman DR. (1994) Signal transduction by lymphocyte antigen receptors. Cell 76: 263-274.

8. Tada T, Ohzeki S, Utsumi K, et al. (1991) Transforming growth factor- $\beta$-induced inhibition of T cell function. J. Immunol. 146: 1077-1082.

9. Young RM, Wright MA, Coogan M, Young ME, Bagash J. (1992) Tumor-derived cytokines induce bone marrow suppressor cells that mediate immunosuppression through transforming growth factor $\beta$. Cancer Immunol. Immunother. 35: 14-18.

10. Malick AP, Garner R, Black SP, Elgert KD. (1984) Normal and tumor-bearing host macrophage factor-mediated modulation of mixedlymphocyte reaction responsiveness: Separation of T-lymphocyte subset susceptibility to enhancing and inhibitory factors. Cell. Immunol. 85: 11 .

11. Walker TM, Burger CJ, Elgert KD. (1994) Tumor growth alters $\mathrm{T}$ cell and macrophage production of and responsiveness to granulocyte-macrophage colony-stimulating factor: Partial dysregulation through interleukin-10. Cell. Immunol. 154: 342-357.

12. Fu Y, Watson G, Jimenez JJ, Wang Y, Lopez DM. (1990) Expansion of immunoregulatory macrophages by granulocyte-macrophage colony-stimulating factor derived from a murine mammary tumor. Cancer Res. 50: 227-234.
13. Parhar RS, Lala PK. (1988) Prostaglandin $\mathrm{E}_{2}$-mediated inactivation of various killer lineage cells by tumor-bearing host macrophages. J. Leukoc. Biol. 44: 474-484.

14. Watson GA, Fu Y, Lopez DM. (1991) Splenic macrophages from tumor-bearing mice coexpressing MAC-1 and MAC- 2 antigens exert immunoregulatory functions via two distinct mechanisms. J. Leukoc. Biol. 49: 126-138.

15. Aoe T, Okamoto Y, Saito T. (1995) Activated macrophages induce structural abnormalities of the $\mathrm{T}$ cell receptor-CD3 complex. $J$. Exp. Med. 181: 1881-1886.

16. Plautz GE, Yang Z, Wu B, Gao X, Huang L, Nabel GJ. (1993) Immunotherapy of malignancy by in vivo gene transfer into tumors. Proc. Natl. Acad. Sci. U.S.A. 90: 4645-4649.

17. Fernandez-Cruz E, Gilman SC, Feldman JD. (1982) Altered levels of mononuclear leukocytes in tumor-bearing rats: decrease of helper $\mathrm{T}$ lymphocytes and increase of suppressor cells. J. Immunol. 129: 1324-1328.

18. Mizoguchi H, O'Shea JJ, Longo DL, Loeffler CM, McVicar DW, Ochoa AC. (1992) Alterations in signal transduction molecules in $\mathrm{T}$ lymphocytes from tumor-bearing mice. Science 258: 1795-1798.

19. Smith DR, Kunkel SL, Burdick MD, et al. (1994) Production of interleukin-10 by human bronchogenic carcinoma. Am. J. Pathol. 145: $18-25$.

20. Howard M, O'Garra A. (1992) Biological properties of interleukin 10. Immunol. Today 13: $198-200$.

21. Ko-Huang L, Lauener RP, Winchester RJ, Geha RS, Vercelli D. (1991) Engagement of CD14 on human monocytes terminates $\mathrm{T}$ cell proliferation by delivering a negative signal to T cells. J. Immunol. 147: $1134-1138$.

22. Stach RM, Rowley DA. (1993) A first or dominant immunization. II. Induced immunoglobulin carries transforming growth factor $\beta$ and suppresses cytolytic $\mathrm{T}$ cell responses to unrelated alloantigens. J. Exp. Med. 178: 841-852.

23. Denham S, Rowland IJ. (1992) Inhibition of the reactive proliferation of lymphocytes by activated macrophages: The role of nitric oxide. Clin. Exp. Immunol. 87: 157-162. 